\title{
Visual attention as a multilevel selection process
}

\author{
SABINE KASTNER and MARK A. PINSK \\ Princeton University, Princeton, New Jersey
}

\begin{abstract}
Natural visual scenes are cluttered and contain many different objects that cannot all be processed simultaneously. Therefore, attentional mechanisms are needed to select relevant and to filter out irrelevant information. Evidence from functional brain imaging reveals that attention operates at various processing levels within the visual system and beyond. First, the lateral geniculate nucleus appears to be the first stage in the processing of visual information that is modulated by attention, consistent with the idea that it may play an important role as an early gatekeeper in controlling neural gain. Second, areas at intermediate cortical-processing levels, such as V4 and TEO, appear to be important sites at which attention filters out unwanted information by means of receptive field mechanisms. Third, the attention mechanisms that operate in the visual system appear to be controlled by a distributed network of higher order areas in the frontal and parietal cortex, which generate top-down signals that are transmitted via feedback connections to the visual system. And fourth, the pulvinar of the thalamus may operate by integrating and coordinating attentional functions in concert with the fronto-parietal network, although much needs to be learned about its functional properties. The overall view that emerges from the studies reviewed in this article is that neural mechanisms of selective attention operate at multiple stages in the visual system and beyond and are determined by the visual processing capabilities of each stage. In this respect, attention can be considered in terms of a multilevel selection process.
\end{abstract}

Natural visual scenes are cluttered and contain many different objects. However, the capacity of the visual system to process information about multiple objects at any given moment in time is limited (e.g., Broadbent, 1958). Hence, attentional mechanisms are needed to select relevant information and to filter out irrelevant information from cluttered visual scenes. Selective visual attention is a broad term that refers to a variety of different behavioral phenomena. Directing attention to a spatial location has been shown to improve the accuracy and speed of subjects' responses to target stimuli that occur at that location (Posner, 1980). Attention also increases the perceptual sensitivity for the discrimination of target stimuli (Lu \& Dosher, 1998), increases contrast sensitivity (Cameron, Tai, \& Carrasco, 2002; Carrasco, Ling, \& Read, 2004), reduces the interference caused by distractors (Shiu \& Pashler, 1995), and improves acuity (Yeshurun \& Carrasco, 1998).

What may be the neural basis underlying these and other behavioral effects of visual attention? There is converging evidence from single-cell physiology studies in nonhuman primates and functional brain mapping studies in humans that selective attention modulates neural activ-

This review elaborates on ideas and parts that were published in Kastner (2004). Support was provided by grants from the NIMH (RO1 MH64043, P50 MH62196) and the Whitehall Foundation to S.K. and by an NSF Graduate Research Fellowship to M.A.P. Correspondence concerning this article should be addressed to S. Kastner, Center for the Study of Brain, Mind, and Behavior, Department of Psychology, Princeton University, Green Hall, Princeton, NJ 08544 (e-mail: skastner@, princeton.edu). ity in the visual system. Attentional response modulation was originally demonstrated in the extrastriate cortex (e.g., Moran \& Desimone, 1985) and has also been shown in the striate cortex, where the modulation appears to depend more on task-related factors, such as the attentional demands of the task at hand or the need to integrate contextual information from areas beyond the classical receptive field (RF; see, e.g., Ito \& Gilbert, 1999; Martinez et al., 1999; Motter, 1993). These findings, in combination with negative results failing to demonstrate attentional modulation at subcortical processing stages, such as the lateral geniculate nucleus (LGN), led to a notion that selective attention affects neural processing only at the cortical level (e.g., Mehta, Ulbert, \& Schroeder, 2000). This notion has recently been revised by demonstrating attentional modulation in the human LGN (O'Connor, Fukui, Pinsk, \& Kastner, 2002). The overall view that appears to emerge from these studies is that neural mechanisms of selective attention operate at multiple stages in the visual system. It is well known that each of the visual processing stages contributes differently to our visual perception. Therefore, it is possible that attentional modulation in the visual system serves different functions at different processing stages, which are determined by the processing capabilities of each stage. In this review, we will discuss some of the evidence in support of such a hypothesis. We will argue that, at the thalamic level, attention may serve to control neural response gain. At early cortical processing stages, attention affects the contextual modulation of neural responses, which may serve important functions in the basic mechanisms of scene segmentation and grouping (Ito \& Gilbert, 1999). At intermediate cortical processing stages, 
attention may mediate the spatial filtering of unwanted information. These multilevel modulatory processes appear to be controlled by a higher order fronto-parietal network of brain areas that may coordinate its function with the pulvinar nucleus of the thalamus. Together, these brain systems cooperate to generate attentional functions that enable us to select relevant visual information for further processing in memory and other cognitive systems to guide actions. In this respect, visual selective attention can be described as a multilevel selection process that operates by integrating information processed in several parallel distributed cortical and subcortical networks. Such a concept is consistent with a vision that Patricia GoldmanRakic expressed in "Topography of Cognition" (1988) for all higher cognitive functions. She wrote: "The picture that emerges ... is that of a highly integrated but distributed (brain) machinery whose resources are allocated to several basic parallel functional systems that bridge all major subdivisions of the cerebrum" (p. 152).

\section{The Lateral Geniculate Nucleus: An Early "Gatekeeper"}

Attentional modulation in the human LGN. The LGN is the thalamic station in the retinocortical projection and has traditionally been viewed as the gateway to the visual cortex (Jones, 1985; Sherman \& Guillery, 2001). It is organized into six layers, each of which receives input from either the contra- or the ipsilateral eye. There are four dorsal LGN layers containing small (parvocellular) neurons that are characterized by sustained discharge patterns and low contrast gain and two ventral layers containing large (magnocellular) neurons that are characterized by transient discharge patterns and high contrast gain (e.g., Merigan \& Maunsell, 1993; Wiesel \& Hubel, 1966).

In addition to retinal afferents, the $\mathrm{LGN}$ receives input from multiple sources, including the striate cortex, the thalamic reticular nucleus (TRN), and the brainstem. The LGN therefore represents the first stage in the visual pathway at which cortical top-down feedback signals could affect information processing. It has proven difficult to study attentional response modulation in the LGN using single-cell physiology, due to the small RF sizes of LGN neurons and the possible confound of small eye movements. Several single-cell physiology studies have failed to demonstrate attentional modulation in the LGN, supporting a notion that selective attention affects neural processing only at the cortical level (e.g., Mehta et al., 2000). We recently revisited the role of the LGN in attentional processing, using fMRI in humans (O'Connor et al., 2002). Functional MRI measures neural activity at a population level that may be better suited to uncover large-scale modulatory activity. Small modulatory effects that cannot be reliably found by measuring neural activity at the single- or multiunit level may be revealed when summed across large populations of neurons.

At the cortical level, selective attention has been shown to affect visual processing in (at least) three different ways. First, neural responses to attended visual stimuli are enhanced relative to the same stimuli when unattended (attentional enhancement; see, e.g., Corbetta, Miezin, Dobmeyer, Shulman, \& Petersen, 1990; Moran $\&$ Desimone, 1985). Second, neural responses to unattended stimuli are attenuated depending on the load of attentional resources engaged elsewhere (attentional suppression; see Rees, Frith, \& Lavie, 1997). And third, directing attention to a location in the absence of visual stimulation and in anticipation of the stimulus onset increases neural baseline activity (attention-related baseline increases; see Kastner, Pinsk, DeWeerd, Desimone, \& Ungerleider, 1999; Luck, Chelazzi, Hillyard, \& Desimone, 1997). We investigated these effects of selective attention in a series of three experiments, which were designed to optimally activate the human LGN. Flickering checkerboard stimuli of high or low contrast were used in all the experiments, which activated the LGN (Chen, Zhu, Thulborn, \& Ugurbil, 1999) and areas in the visual cortex, including V1, V2, ventral and dorsal V3, V4, TEO, $\mathrm{V} 3 \mathrm{~A}$, and MT/MST (referred to as MT), as determined on the basis of retinotopic mapping (Kastner et al., 2001; Sereno et al., 1995).

To investigate attentional response enhancement in the LGN, checkerboard stimuli were presented to the left or the right hemifield while subjects directed attention to the stimulus (attended condition) or away from the stimulus (unattended condition). In the unattended condition, attention was directed away from the stimulus by having the subjects count letters at fixation. The letter-counting task ensured proper fixation and prevented the subjects from covertly attending to the checkerboard stimuli (Kastner, DeWeerd, Desimone, \& Ungerleider, 1998). In the attended condition, the subjects were instructed to covertly direct attention to the checkerboard stimulus and to detect luminance changes that occurred randomly in time at $10^{\circ}$ eccentricity. In our statistical model, stimulation of the left visual hemifield was contrasted with stimulation of the right visual hemifield. Thereby, the analysis was restricted to voxels activated by the peripheral checkerboard stimuli and excluded foveal stimulus representations. Relative to the unattended condition, the neural activity evoked by both the high-contrast stimulus and the low-contrast stimulus increased significantly in the attended condition (Figure 1A). The attentional response enhancement was shown to be spatially specific. These results suggest that attention facilitates visual processing in the LGN by enhancing neural responses to an attended stimulus, relative to those evoked by the same stimulus when ignored.

To investigate attentional load dependent suppression in the LGN, high- and low-contrast checkerboard stimuli were presented to the left or the right hemifield while the subjects performed either an easy attention task or a hard attention task at fixation and ignored the peripheral checkerboard stimuli. During the easy attention task, the subjects counted infrequent, brief color changes of the fixation cross. During the hard attention task, the sub- 


\section{Lateral Geniculate Nucleus}

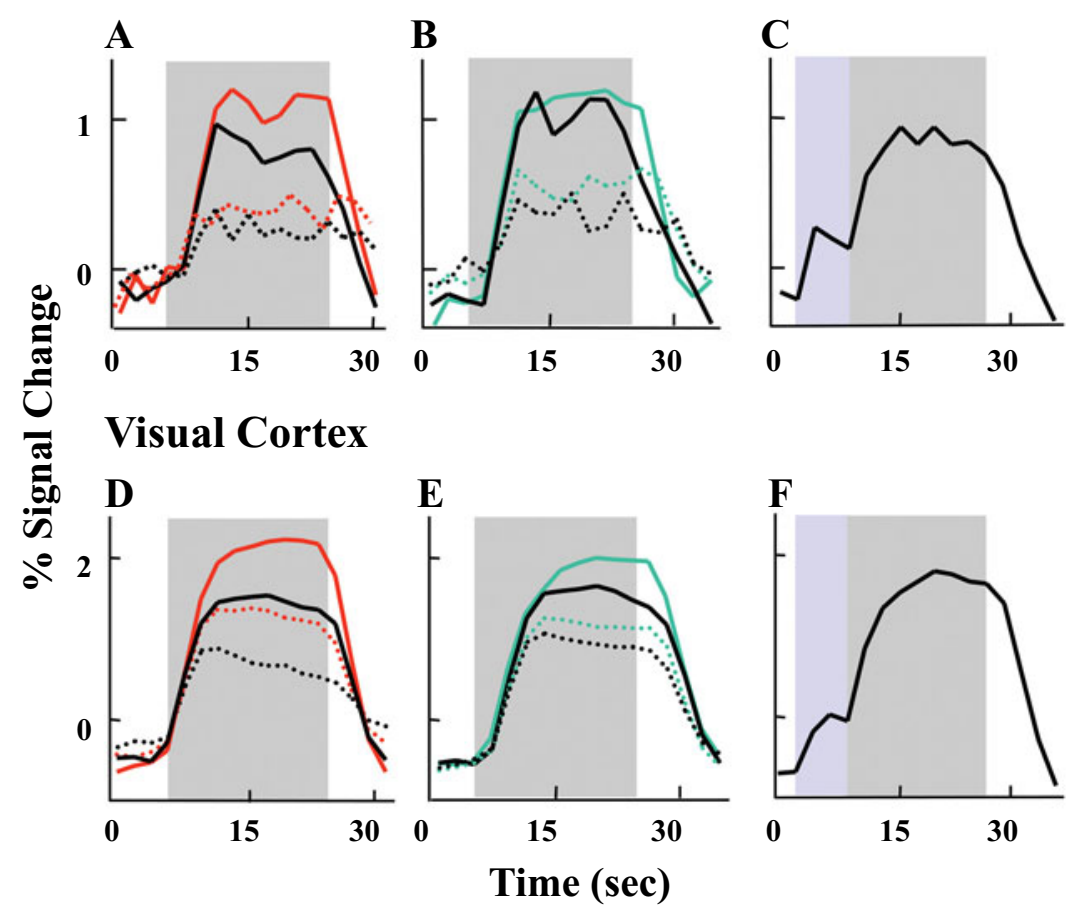

Figure 1. Time series of fMRI signals in the lateral geniculate nucleus (LGN) and in the visual cortex: group analysis $(n=4)$. Data from the LGN and the visual cortex were combined across left and right hemispheres. Activity in the visual cortex was pooled across areas V1, V2, V3/VP, V4, TEO, V3A, and MT/MST. (A, D) Attentional enhancement. During directed attention to the stimuli (red curves), responses to both the high-contrast stimulus $(100 \%$, solid curves) and the low-contrast stimulus $(5 \%$, dashed curves) were enhanced relative to an unattended condition (black curves). (B, E) Attentional suppression. During an attentionally demanding hard fixation task (black curves), responses evoked by both the high-contrast stimulus $(100 \%$, solid curves) and the low-contrast stimulus $(10 \%$, dashed curves) were attenuated relative to an easy attention task at fixation (green curves). (C, F) Baseline increases. Baseline activity was elevated during directed attention to the periphery of the visual hemifield, in expectation of the stimulus onset (blue). Gray shades indicate the checkerboard presentation periods. From "Attention Modulates Responses in the Human Lateral Geniculate Nucleus," by D. H. O'Connor, M. M. Fukui, M. A. Pinsk, and S. Kastner, 2002, Nature Neuroscience, 5, p. 1206. Copyright 2002 by the Nature Publishing Group. Reprinted with permission.

jects counted letters at fixation. Behavioral performance was $99 \%$ correct, on average, in the easy attention task and $54 \%$ correct in the hard attention task, thus indicating the differences in attentional demands. Relative to the easy task condition, neural activity evoked by the highand the low-contrast stimuli decreased significantly in the hard task condition (Figure 1B). This finding suggests that neural activity evoked by ignored stimuli was attenuated in the LGN, depending on the load of attentional resources engaged elsewhere.

To investigate attention-related baseline increases in the LGN, the subjects were cued to covertly direct attention to the periphery of the left or the right visual hemifield and to expect the onset of the stimulus. The expectation period was followed by attended presentations of a high-contrast checkerboard stimulus, during which the subjects counted the occurrence of luminance changes. During the expectation period, fMRI signals increased significantly, relative to the preceding blank period in which the subjects were fixating but not directing attention to the periphery. Because the visual input, a gray blank screen, was identical in both conditions, the increase in baseline activity appeared to be related to directed attention and may be interpreted as a bias in favor of the attended location (see also the Frontal and Parietal Cortex: Sorting Out Sources and Sites section). The baseline increase was followed by a further response increase evoked by the visual stimuli (Figure 1C). It is important to note that, because of our statistical model, the increase in baseline activity was not related to the cue, which was presented at fixation. This finding suggests that neural activity in the LGN can be affected by attention- 
related top-down signals even in the absence of any visual stimulation whatsoever.

In summary, these studies indicate that selective attention modulates neural activity in the LGN by enhancing neural responses to attended stimuli, by attenuating those to ignored stimuli, and by increasing baseline activity in the absence of visual stimulation.

Comparison of attention effects in the LGN and the visual cortex. At the cortical level, qualitatively similar effects of attention were found, as shown in the time
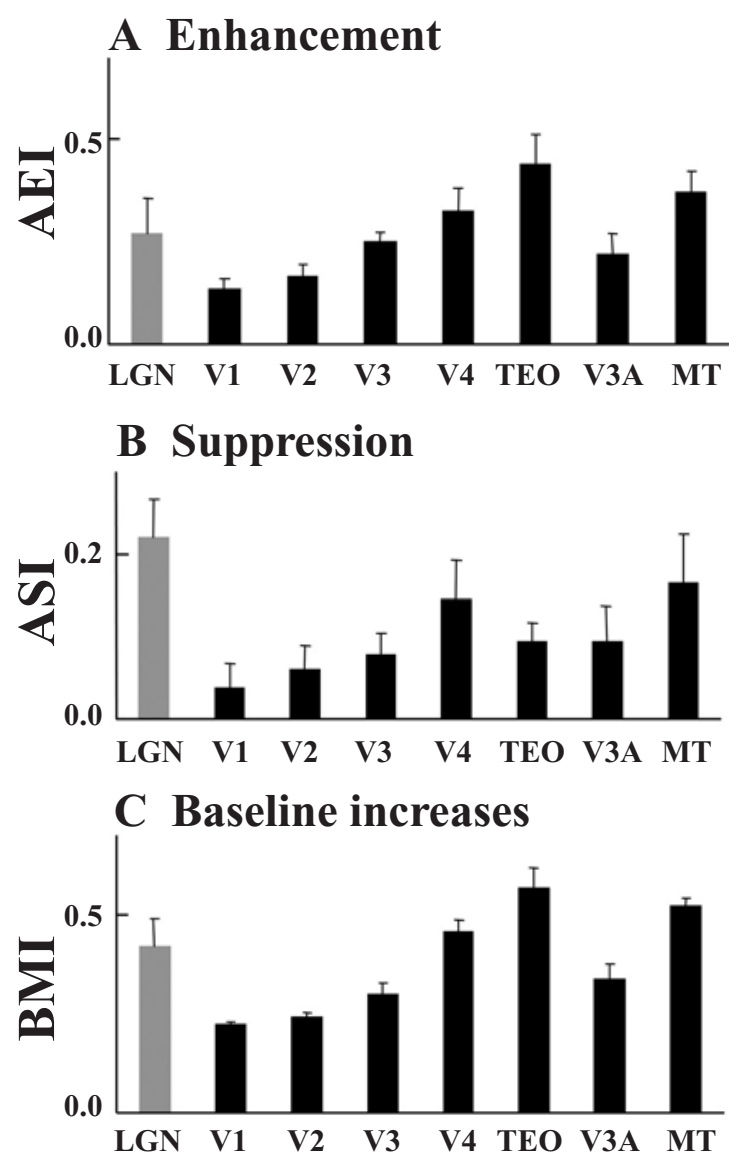

Figure 2. Attentional response modulation in the lateral geniculate nucleus (LGN) and in the visual cortex. Attention effects that were obtained in the experiments presented in Figure 1 were quantified by defining several indices: (A) the attentional enhancement index (AEI), (B) the attentional suppression index (ASI), and (C) the baseline modulation index (BMI). For all indices, larger values indicate larger effects of attention. Index values were computed for each subject on the basis of normalized and averaged signals obtained in the different attention conditions and are presented as averaged index values from 4 subjects (for index definitions, see O'Connor, Fukui, Pinsk, \& Kastner, 2002). In the visual cortex, attention effects increased from early to later processing stages. Attention effects in the LGN were larger than those in V1. Vertical bars indicate $S E M$ across subjects. From "Attention Modulates Responses in the Human Lateral Geniculate Nucleus," by D. H. O'Connor, M. M. Fukui, M. A. Pinsk, and S. Kastner, 2002, Nature Neuroscience, 5, p. 1206. Copyright 2002 by the Nature Publishing Group. Reprinted with permission. series of fMRI signals averaged across all the activated areas in the visual cortex - that is, areas V1, V2, V3, V4, TEO, V3A, and MT (Figures 1D-1F). The attention effects found at the thalamic and at the cortical level were compared by normalizing the mean fMRI signals evoked in the LGN and in each activated cortical area and by computing index values for each attention effect and each area, which are measures of the magnitude of a given attention effect. This analysis is shown in Figure 2; larger index values indicate larger effects of attention. It should be noted that index values cannot be easily compared across attention effects, due to differences in index definitions and attention tasks. In accordance with previous findings (Cook \& Maunsell, 2002; Kastner et al., 1998; Martinez et al., 1999; Mehta et al., 2000), the magnitude of all attention effects increased from early to more advanced processing levels along both the ventral and the dorsal pathways of the visual cortex (Figures 2A$2 \mathrm{C})$. This is consistent with the idea that attention operates through top-down signals that are transmitted via corticocortical feedback connections in a hierarchical fashion. Thereby, areas at advanced levels of visual cortical processing are more strongly controlled by attention mechanisms than are early processing levels. This idea is supported by single-cell recording studies, which have shown that attention effects in area TE of the inferior temporal cortex have a latency of approximately $150 \mathrm{msec}$ (Chelazzi, Duncan, Miller, \& Desimone, 1998), whereas attention effects in V1 have a longer latency of approximately 230 msec (Roelfsema, Lamme, \& Spekreijse, 1998). According to this account, one would predict smaller attention effects in the LGN than in the striate cortex. Surprisingly, it was found that all attention effects tended to be larger in the LGN than in the striate cortex (Figures 2A-2C). This finding suggests that attentional response modulation in the LGN is unlikely to be due solely to corticothalamic feedback from the striate cortex but may be further influenced by additional sources of input (see below). Other possibilities that may explain the differences in magnitude of the modulation between the LGN and V1 include regional disparities underlying the blood-oxygenation-level-dependent (BOLD) signal or nonlinearities in thalamocortical signal transmission. Furthermore, it is possible that differences in strength of attention effects at different processing stages may reflect the degree to which multiple parallel inputs converge on a given area, rather than a feedback mechanism that reverses the processing hierarchy.

Sources of modulatory influences on the LGN. The findings reviewed thus far challenge the classical notion that attention effects are confined to cortical processing. Furthermore, they suggest the need to revise the traditional view of the LGN as a mere gateway to the visual cortex. In fact, due to its afferent input, the LGN may be in an ideal strategic position to serve as an early gatekeeper in attentional gain control. In addition to corticothalamic feedback projections from V1, which comprise about $30 \%$ of its modulatory input, the $\mathrm{LGN}$ receives 
another $30 \%$ of modulatory inputs from the TRN (Sherman \& Guillery, 2002). For several reasons, the TRN has long been implicated in theoretical accounts of selective attention (Crick, 1984). First, all feedforward projections from the thalamus to the cortex, as well as their reverse projections, pass through the TRN. Second, the TRN receives inputs not only from the LGN and V1, but also from several extrastriate areas and the pulvinar. Thereby, it may serve as a node where several cortical areas and thalamic nuclei of the visual system can interact to modulate thalamocortical transmission through inhibitory connections to LGN neurons (Guillery, Feig, \& Lozsádi, 1998; see also Figure 7). And third, the TRN contains topographically organized representations of the visual field and can thereby modulate thalamocortical or corticothalamic transmission in spatially specific ways. Similarly, all corticofugal projections are organized in topographic order. Other modulatory influences on the LGN stem from the parabrachial nucleus of the brainstem. These cholinergic projections, another $30 \%$ of the modulatory input to the LGN, are more diffusely organized (Erisir, Van Horn, \& Sherman, 1997), which makes a possible role in spatially selective attention more difficult to account for.

Cortical, reticular, and brainstem influences appear to control two different response modes of LGN neurons, known as burst and tonic firing, both occurring in the awake state (Sherman, 2001). In both modes, information is relayed to the cortex, but with different content and consequences. In the tonic mode, the retinal input to LGN neurons is relayed faithfully and almost linearly to the cortex, thereby providing most detailed information for reconstructing visual scenes. In the burst mode, neurons respond with a transient all-or-nothing response to incoming visual information, which leads to considerable distortion and nonlinearity in the relay of information from the retina to the cortex. However, spontaneous neural activity is much diminished in the burst mode, thereby enhancing signal-to-noise ratios and signal detectability. Therefore, the burst mode may provide the functionality to efficiently detect new and salient information in the environment, whereas the tonic mode would strengthen the faithful transmission of this information to the cortex for more detailed analyses. Activation of cortical and brainstem afferents to LGN neurons appear to control the tonic mode, whereas the reticular afferents control the burst mode.

The different response modes of LGN neurons that are promoted by the main modulatory sources of input to the LGN provide potential neural correlates for Crick's (Crick, 1984) attention theory. However, it is still hypothetical whether switches in response modes reflect different attentional states or selection mechanisms. In particular, it is unclear how the diffuse projections of the brainstem might account for controlling topographically organized, selective neural population responses. Furthermore, it is not likely that the attentional modulation in the human LGN, found with fMRI (O'Connor et al., 2002), is related to switches between response modes due to the poor temporal resolution of brain-imaging techniques. Rather, this modulation may reflect an attentional gain control mechanism that is exerted when a visual stimulus is selected for further processing and the information is relayed in a more linear fashion.

In summary, the LGN appears to be the first stage in the processing of visual information that is modulated by attentional top-down signals. Much remains to be learned about the complex thalamic circuitry that may subserve attentional functions related to the different response modes and to the control of neural gain in the LGN.

Possible nature of attentional gain control mechanism. What may be the nature of the gain mechanism that is controlled by attention at the level of the LGN? One possibility is that selective attention simply increases the responsivity of LGN neurons in a multiplicative fashion, thereby increasing neural signals evoked by visual stimuli, relative to background noise (spontaneous activity), or by enhancing synaptic efficiency to process upcoming stimuli during stimulus anticipation. Evidence in support of a multiplicative attentional gain control mechanism has been found in the monkey and the human extrastriate cortex (McAdams \& Maunsell, 1999; Murray $\&$ Wojciulik, 2004). It has been shown in physiology studies that responses evoked by an attended preferred stimulus (e.g., an optimally oriented bar) were enhanced more strongly than those evoked by an attended nonpreferred stimulus. A second possibility is that selective attention increases neural gain dependent on stimulus contrast. Attention has been shown to increase contrast sensitivity in V4 neurons by enhancing neural responses evoked by low-contrast stimuli more strongly relative to those evoked by high-contrast stimuli (Reynolds, Pasternak, \& Desimone, 2000). This result can be interpreted as an increase in effective stimulus contrast by attention. In accord with these neurophysiological findings, it has been shown in behavioral studies that attention decreases contrast thresholds and changes the perceived strength of a stimulus, thereby altering its appearance (Cameron et al., 2002; Carrasco et al., 2004). A third possibility is that attention influences the magnocellular and parvocellular neural systems at the level of the LGN differently, as has been suggested by Vanduffel, Tootell, and Orban (2000). In their deoxyglucose study, attention-dependent changes in metabolic activity were shown only for the magnocellular, but not for the parvocellular, LGN layers. This result has been corroborated by behavioral and recent neuroimaging studies suggesting a selective modulation of the magnocellular system by attention (Cheng, Eysel, \& Vidyasagar, 2004; Schneider, \& Kastner, 2004; Steinman, Steinman, \& Lehmkuhle, 1997). Importantly, due to the sensitivity of the magnocellular system to stimulus contrast, which is 10 times higher than that of the parvocellular system, a selective modulation of the magnocellular system may explain the attention effects on contrast gain found at the cortical level. It should be noted that none of these possibilities are mutually exclu- 
sive. Future investigations will be necessary to explore the nature of attentional gain control in the human LGN further.

\section{Visual Cortex: Spatial Filtering of Distractor Information in Areas V4 and TEO}

At the thalamic level, attention may serve to amplify gain, thereby increasing neural signals relative to background noise. At the cortical level, one important function of attention is to filter out another type of noise that is induced by the vast majority of visual information: the unwanted information from distractors (Reynolds \& Desimone, 1999). Evidence from single-cell physiology and lesion studies in monkeys and fMRI studies in humans suggests that areas V4 and TEO are important sites where relevant information is selected and irrelevant information is filtered out (DeWeerd, Peralta, Desimone, \& Ungerleider, 1999; Kastner et al., 1998; Reynolds, Chelazzi, \& Desimone, 1999). In this section, we will first describe the evidence that multiple stimuli in cluttered visual scenes compete for neural representation in the visual cortex. And second, we will describe intraand extra-RF mechanisms for the spatial filtering of unwanted information.

A neural basis of competition among multiple stimuli. Due to the limited processing capacity of the visual system, multiple objects present at the same time in visual scenes appear to compete for neural representation. What is the neural basis for competitive interactions among multiple objects in the visual field? In single-cell physiology studies, neural responses to a single visual stimulus presented alone in a neuron's RF were compared with the responses evoked by the same stimulus when a second one was presented simultaneously within the same RF. The responses to the paired stimuli were found to be smaller than the sum of the responses evoked by each stimulus individually and turned out to be a weighted average of the individual responses (Reynolds et al., 1999). This result suggests that multiple stimuli present at the same time within a neuron's RF are not processed independently but interact with each other in a mutually suppressive way, indicating competition for neural representation. Competitive interactions among multiple stimuli present at the same time in the visual field have been found in several areas, including V2, V4, MT, MST, and IT (Miller, Gochin, \& Gross, 1993; Recanzone, Wurtz, \& Schwarz, 1997; Reynolds et al., 1999).

On the basis of hypotheses derived from these monkey physiology studies, competitive interactions among multiple stimuli were investigated in the human cortex, using fMRI (Kastner et al., 1998; Kastner et al., 2001). Colorful visual stimuli, which optimally activate the ventral visual cortex, were presented to the periphery of the visual field in four nearby locations while subjects performed a letter-counting task at fixation. The stimuli were presented under two different presentation conditions, sequential and simultaneous. In the sequential presentation condition, a single stimulus appeared in one of the four locations, then another appeared in a different location, and so on, until each of the four stimuli had been presented in the different locations. In the simultaneous presentation condition, the same four stimuli appeared in the same four locations, but they were presented together. Thus, integrated over time, the physical stimulation parameters were identical in each of the four locations in the two presentation conditions. However, suppressive (competitive) interactions among stimuli within RFs could take place only in the simultaneous, not in the sequential, presentation condition. On the basis of the results from monkey physiology, it was predicted that the fMRI signals would be smaller during the simultaneous than during the sequential presentation condition, due to the presumed mutual suppression induced by the competitively interacting stimuli.

In the ventral visual cortex, areas V1, V2, V4, and TEO were consistently activated during visual stimulation, as compared with blank periods. As predicted by our hypothesis, simultaneous presentations evoked weaker responses than sequential presentations did in all the activated visual areas. The response differences were smallest in V1 (Figure 3A) and increased in magnitude toward ventral extrastriate areas V4 (Figure 3A) and TEO. This increase in magnitude of the suppression effects across visual areas suggested that the competitive interactions were scaled to the increase in the RF size of the neurons within these areas. That is, the small RFs of the neurons in V1 and V2 would encompass only a small portion of the visual display, whereas the larger RFs of the neurons in V4 and TEO would encompass all four stimuli. Therefore, suppressive interactions among the stimuli within RFs could take place most effectively in these more anterior extrastriate visual areas. In V1 and V2, it is likely that surround inhibition from regions outside the classical RF contributed to the small sensory suppression effects (e.g., Kastner, Nothdurft, \& Pigarev, 1999). To rule out the possibility that the differential responses evoked by the two presentation conditions reflected differences in the rate of transient stimulus onsets, suppressive interactions were also demonstrated in a control experiment, in which the presentation rate was kept constant (see Kastner et al., 1998; Kastner et al., 2001).

The idea that suppressive interactions are scaled to RF size was tested directly in a second study, in which the spatial separation among the stimuli was increased (Kastner et al., 2001). According to the RF hypothesis, the magnitude of the suppressive interactions should be inversely related to the degree of spatial separation among the stimuli. In agreement with this idea, separating the stimuli by $4^{\circ}$ abolished suppressive interactions in V2, reduced them in V4, but did not affect them in TEO. Separating the stimuli by $6^{\circ}$ led to a further reduction of suppression effects in V4 but again had no effect in TEO. These results confirmed our hypothesis that competitive interactions occur mainly at the level of the RF. Furthermore, by systematically varying the spatial separation among the stimuli and measuring the magnitude of sup- 


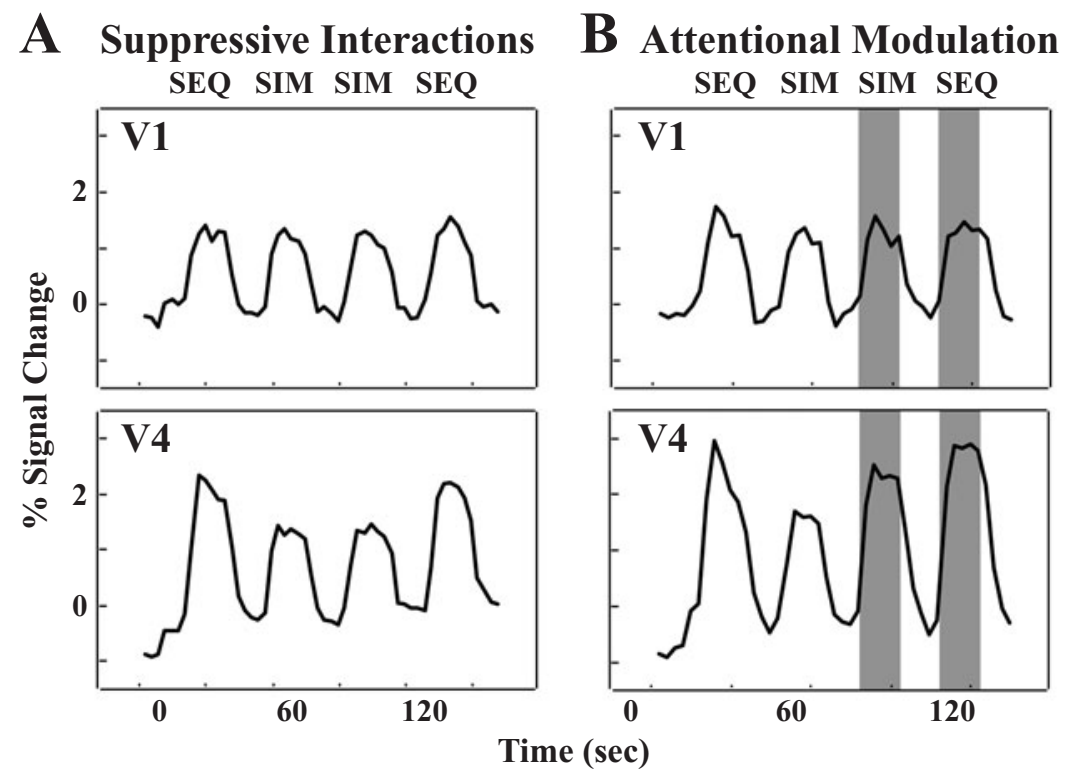

\begin{abstract}
Figure 3. Competitive interactions and attentional modulation in the visual cortex. (A) Suppressive interactions in V1 and V4. Simultaneously presented stimuli evoked less activity than did sequentially presented stimuli in V4, but not in V1, suggesting that suppressive interactions were scaled to the receptive field size of neurons in the visual cortex. (B) Attentional modulation of suppressive interactions. The suppression effect in V4 was replicated in the unattended condition of this experiment, when the subjects' attention was directed away from the stimulus display (unshaded time series). Spatially directed attention (shaded time series) increased responses to simultaneously presented stimuli to a larger degree than to sequentially presented ones in V4. From "Mechanisms of Directed Attention in the Human Extrastriate Cortex as Revealed by Functional MRI," by S. Kastner, P. DeWeerd, R. Desimone, and L. G. Ungerleider, 1998, Science, 282, p. 109. Copyright 1998 by the American Association for the Advancement of Science. Adapted with permission.
\end{abstract}

pressive interactions, we estimated the average RF sizes at an eccentricity of about $5^{\circ}$ to be less than $2^{\circ}$ in V1, in the range of $2^{\circ}-4^{\circ}$ in $\mathrm{V} 2$, about $6^{\circ}$ in $\mathrm{V} 4$, and larger than $6^{\circ}$ but still confined to a quadrant in TEO. These numbers may underestimate RF sizes in the human visual cortex, due to additional suppressive influences from beyond the RF, which cannot be distinguished from interactions within RFs in our experimental paradigm. It was striking, however, that these estimates of RF sizes in the human visual cortex, as determined on the basis of hemodynamic responses, are similar to those measured in the homologous visual areas of monkeys, as defined at the level of single cells (e.g., Desimone \& Ungerleider, 1989).

In summary, these fMRI studies have begun to establish a neural basis for competition among multiple stimuli present at the same time in the visual field. Importantly, the degree to which this competition occurs appears to critically depend on the RF sizes of neurons across visual cortical areas. This has important implications for the operations of spatial selective attention in the visual cortex, as will be described in the next section.

Spatial filtering of distractor information at the level of the receptive field. In single-cell recording studies, it has been demonstrated that spatially directed attention can influence the competition among multiple stimuli in favor of one of the stimuli by modulating competitive interactions. When a monkey directed attention to one of two competing stimuli within an RF, the responses in extrastriate areas V2, V4, and MT were as large as those to that stimulus presented alone, thereby eliminating the suppressive influence of the competing stimulus (Recanzone \& Wurtz, 2000; Reynolds et al., 1999). The attention effects were less pronounced when the second stimulus was presented outside the RF, suggesting that competition for processing resources within visual cortical areas takes place most strongly at the level of the RF. These findings imply that attention may resolve the competition among multiple stimuli by counteracting the suppressive influences of nearby stimuli, thereby enhancing information processing at the attended location. This may be an important mechanism by which attention filters out information from nearby distractors (Desimone \& Duncan, 1995; Duncan, 1996).

A similar mechanism appears to operate in the human visual cortex (Kastner et al., 1998). We studied the effects of spatially directed attention on multiple competing visual stimuli in a variation of the paradigm described in the last section. In addition to the two different presentation conditions, sequential and simultaneous, two differ- 
ent attention conditions were tested, attended and unattended. During the unattended condition, attention was directed away from the peripheral visual display by having the subjects count letters at fixation. In the attended condition, the subjects were instructed to attend covertly to the peripheral stimulus location closest to fixation in the display and to count the occurrences of one of the four stimuli. On the basis of the results from monkey physiology, we predicted that attention should reduce suppressive interactions among stimuli. Thus, responses evoked by the competing, simultaneously presented stimuli should be enhanced more strongly than responses evoked by the noncompeting sequentially presented stimuli.

Directing attention to the location closest to fixation in the display enhanced activity to sequentially and to simultaneously presented stimuli in extrastriate areas V2/VP, $\mathrm{V} 4$, and TEO, with increasing effects from early to later stages of visual processing; attention effects were absent in V1 with this experimental paradigm (Figure 3B). In accordance with our prediction, directed attention led to greater increases of fMRI signals to simultaneously presented stimuli than to sequentially presented stimuli in areas V4 (Figure 3B) and TEO. The magnitude of the attention effect scaled with the magnitude of the suppressive interactions among stimuli, with the strongest reduction of suppression occurring in ventral extrastriate areas V4 (Figure 3B) and TEO, suggesting that the effects scaled with RF size. These findings support the idea that directed attention enhances the information processing of stimuli at the attended location by counteracting suppression induced by nearby stimuli, which compete for limited processing resources, thereby filtering out unwanted information from nearby distractors.

This filter mechanism is compatible with the idea that directed attention to a stimulus may cause the RF to shrink around the attended stimulus, thereby leaving the unattended stimuli in nearby locations outside the RF (Moran \& Desimone, 1985; Reynolds \& Desimone, 1999). Given that the magnitude of suppressive interactions scaled with RF size in our fMRI studies (Kastner et al., 2001), we estimated the RF sizes in V4 and TEO during directed attention to the display. The reduced suppressive interactions in V4 and TEO during directed attention were similar in magnitude to the suppressive interactions obtained in area V2 in the unattended condition. Hence, it is possible that directed attention caused a constriction of RFs in V4 and TEO from $4^{\circ}-8^{\circ}$ to about $2^{\circ}$, thereby presumably enhancing spatial resolution. This interpretation is compatible with behavioral studies showing that spatial attention improves acuity (Yeshurun \& Carrasco, 1998).

In summary, areas at intermediate levels of visual processing, such as V4 and TEO, appear to be important sites for the filtering of unwanted information by counteracting competitive interactions among stimuli at the level of the RF. This notion has also been supported by studies in a patient with an isolated V4 lesion and in monkeys with lesions of areas V4 and TEO (DeWeerd et al., 1999; Gal- lant, Shoup, \& Mazer, 2000). In these studies, subjects performed an orientation discrimination of a grating stimulus in the absence and in the presence of surrounding distractor stimuli. Significant performance deficits were observed in the distractor-present, but not in the distractorabsent, condition, suggesting a deficit in the efficacy of the filtering of distractor information.

The neural fate of distractor information. The studies described thus far have provided intriguing evidence that selective attention operates by modulating competitive interactions among multiple stimuli for neural representation at the level of the RF. Given the RF sizes of neurons in areas V4 and TEO, this attention mechanism may serve to filter out distractors from nearby locations at a local scale of $4^{\circ}-8^{\circ}$. Importantly, this mechanism provides a neural correlate for an attentional spotlight that operates across circumscribed regions of visual space (Brefczynski \& DeYoe, 1999; Eriksen \& St. James, 1986; Tootell et al., 1998). The size of the spotlight may be flexibly scaled to the different RF sizes of early and intermediate visual areas.

Less is known about the neural representation of unattended stimuli outside the focus of attention. Results from neuroimaging and behavioral studies suggest that the processing of unattended stimuli depends on the degree to which attentional resources are engaged by an attended stimulus (Lavie \& Tsal, 1994; Rees et al., 1997; Yi, Woodman, Widders, Marois, \& Chun, 2004). For example, Rees et al. (1997) demonstrated that activation in area MT evoked by unattended moving stimuli was abolished when subjects performed a linguistic task of high attentional load, relative to a low-load version of the task at fixation. In contrast, results from patients suffering from visuospatial hemineglect have shown that neural responses evoked by faces and object stimuli presented to the neglected hemifield were similar, as compared with those evoked by the same stimuli presented to the intact hemifield (Rees et al., 2000; Vuilleumier et al., 2001), suggesting that unattended stimuli undergo processing to advanced stages of category-specific object representations.

We have investigated the neural fate of unattended stimuli in an fMRI study in which attended (target) stimuli were presented to the periphery of the upper right quadrant while ignored (distractor) stimuli were presented to a corresponding location in the contralateral hemifield (Pinsk, Doniger, \& Kastner, 2004). Thereby, neural activity evoked by target and distractor stimuli could be dissociated in visual areas with a quadrant or hemifield representation within the spatial resolution limits of fMRI. Target stimuli evoked activity in the left visual cortex, whereas distractor stimuli evoked activity in the right visual cortex. The subjects covertly directed attention to a series of sequentially presented target stimuli and performed either a low attentional load or a high attentional load search task while irrelevant distractor stimuli appeared in the contralateral hemifield. In both search tasks, the stimuli were identical but appeared in a 


\section{Target-Related Distractor-Related Activity}

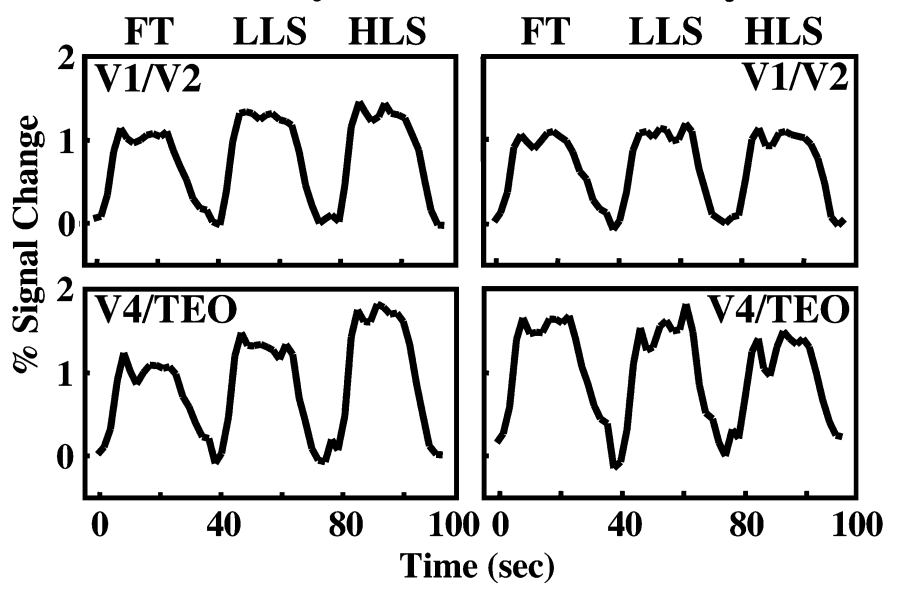

Figure 4. Attentional facilitation and suppression in the visual cortex. Time series of fMRI signals for target search related activity and distractor-related activity in combined areas V1/V2 and V4/TEO, averaged across scans and subjects. Time courses are shown during the fixation task, when both stimuli were ignored (FT), and during a low-load (LLS) and a high-load search (HLS) task. In V1 and V2, target-related activity was enhanced for attended, relative to fixation, conditions to a similar degree during the two search task conditions. Distractor-related activity was not affected by the load manipulation. Conversely, in V4 and TEO, target-related activity was greater during the high-load, as compared with the low-load, search condition; distractor-related activity was suppressed depending on attentional load. From "Push-Pull Mechanism of Selective Attention in Human Extrastriate Cortex," by M. A. Pinsk, G. M. Doniger, and S. Kastner, 2004, Journal of Neurophysiology, 92, p. 625. Copyright 2004 by the American Physiological Society. Reprinted with permission.

different sequence. Differences in attentional load between the two search tasks were established in behavioral studies. For the target search related activity, we predicted that neural activity should increase with increasing attentional load (Ress et al., 2000; Spitzer \& Richmond, 1991). For the distractor-related activity, the prediction was less clear, and several different possibilities needed to be considered. First, neural responses evoked by distractor stimuli may be suppressed, depending on the attentional load of the task, as was previously shown in the dorsal extrastriate cortex (Rees et al., 1997). Second, neural responses evoked by distractor stimuli in the contralateral hemifield may not be affected by attention, as has been suggested by studies of patients suffering from visuospatial hemineglect. In these patients, neural responses evoked by object stimuli presented to the neglected hemifield were similar, as compared with those evoked by the same stimuli presented to the intact hemifield (Rees et al., 2000; Vuilleumier et al., 2001). And third, both mechanisms may operate at different processing levels.

Target and distractor stimuli consistently evoked activity in areas V1, V2, V4, and TEO of the left and the right hemispheres, respectively, relative to interleaved blank periods. In (left) V1 and V2, target search related activity was enhanced to a similar degree during the high-load and the low-load search tasks, relative to a control condition during which the subjects ignored target stimuli and counted letters at fixation (FT, fixation task; Figure 4). In (left) V4 and TEO, target search related activity was also enhanced relative to the control condition, but the attentional response enhancement increased with increasing attentional load, as predicted by our hypothesis (LLS, low load search; HLS, high load search; Figure 4). In these areas, fMRI signals evoked by target stimuli reflected the subjects' behavioral performance. In (right) V1 and V2, distractor-related activity was not affected, suggesting that visual processing was not controlled by attention and was mediated by bottomup mechanisms (Figure 4) in accordance with the results from neglect patients. In (right) V4 and TEO, however, distractor-related activity was suppressed depending on the attentional load (Figure 4), indicating that attentional load dependent suppression operates not only in the dorsal, but also in the ventral extrastriate cortex. Thus, in areas at intermediate processing stages, the attentional load dependent suppression of distractor-related activity mirrored the attentional load dependent enhancement of 
target search related activity. This finding presents direct evidence for a long-ranging and load-dependent pushpull mechanism of selective attention that enhances neural activity evoked by selected stimuli and suppresses activity evoked by unattended stimuli operating at intermediate processing stages of the visual cortex. This mechanism may serve to partially filter out unwanted information over large spatial scales.

Notably, the suppressive mechanism operated over large portions, if not the entire, visual scene, suggesting that the focus of attentional selection may be surrounded by extensive suppressive zones (LaBerge \& Brown 1989; Smith, Singh, \& Greenlee, 2000; Tsotsos, Culhane, \& Cutzu, 2001; Vanduffel et al., 2000). This result may provide a neural basis for psychophysical studies that have implicated suppressive surrounds around the locus of attentional selection on the basis of poorer performance in discriminating and longer reaction times in responding to distractor stimuli (Bahcall \& Kowler, 1999; Caputo \& Guerra, 1998; Cave \& Zimmerman, 1997; Cutzu \& Tsotsos, 2003). How may such a long-ranging mechanism be implemented at the neural level? There are several possibilities that need to be considered. First, visual stimuli presented to the contralateral hemifield have been shown to influence the processing of stimuli within RFs of the ipsilateral hemifield in area V4 (Desimone, Moran, Schein, \& Mishkin, 1993). Such longranging and context-dependent extra-RF modulation of neural responses is thought to be mediated by transcallosal connections in this area (Van Essen, Newsome, \& Bixby, 1982). Selective attention may operate in the visual cortex by affecting local circuits that mediate context modulation, as has previously been shown in V1 (Ito \& Gilbert, 1999). Second, it has been demonstrated that microstimulation of the frontal eye field (FEF) can enhance neural activity in area V4 at target locations and suppress activity at distractor locations (Moore \& Armstrong, 2003). Thus, the attention effects obtained in the extrastriate cortex in our study may be under feedback control of higher order areas of a fronto-parietal attention network that mediate both target selection and distractor suppression (Everling, Tinsley, Gaffan, \& Duncan, 2002; Schall \& Thompson, 1999), as further described in the Frontal and Parietal Cortex: Sorting out Sources and Sites section. And third, results from computational neuroscience have shown that long-ranging suppressive mechanisms may result from feedback between visual areas flowing from higher to lower areas (Tsotsos et al., 2001). It should be noted that these possibilities are not mutually exclusive.

In summary, our findings present evidence for a loaddependent push-pull mechanism of selective attention that operates in areas V4 and TEO by facilitating responses to attentionally selected stimuli and by suppressing responses to ignored stimuli at the same time. Such a mechanism may mediate the filtering of unwanted information over large spatial scales.

A push-pull model of spatial attention. The studies described in the last two sections suggest that selective attention operates in areas V4 and TEO by using intraand extra-RF mechanisms to filter out the vast majority of information from visual scenes: the unattended stimuli. At the level of the RF, attention appears to operate by counteracting competitive (suppressive) influences from nearby distractor stimuli; beyond the RF, suppressive mechanisms of attention appear to range over large portions of, if not the entire, visual scene. These intra- and extra-RF mechanisms for the spatial filtering of unwanted information can be combined into a unified framework, a push-pull model of spatially selective attention, as depicted schematically in Figure 5. We assume that the facilitatory and the suppressive attentional mechanisms are interdependent - that is, activation of the facilitatory mechanism will also lead to an activation of the suppressive mechanism at the level of the RF and beyond. The suppressive mechanisms occur most strongly at the level of the RF and are more moderate beyond the RF, decreasing gradually with increasing distance from the locus of attentional selection. The lateral extent of suppressive mechanisms that filter out irrelevant information depends on the strength with which the facilitatory mechanism is activated. The overall activation of this push-pull mechanism will depend on the attentional demands of the task at hand (Figure 5). Even though this model is certainly an oversimplification of the underlying mechanisms, it provides a useful framework to explain a number of empirical findings. First, attention effects are typically smaller at early than at later stages of processing (e.g., O'Connor et al., 2002). This result is often taken as evidence for a top-down attentional feedback mechanism that uses cortico-cortical feedback projections and basically reverses the processing hierarchy

\section{Extra-RF Surround RF Level Surround}

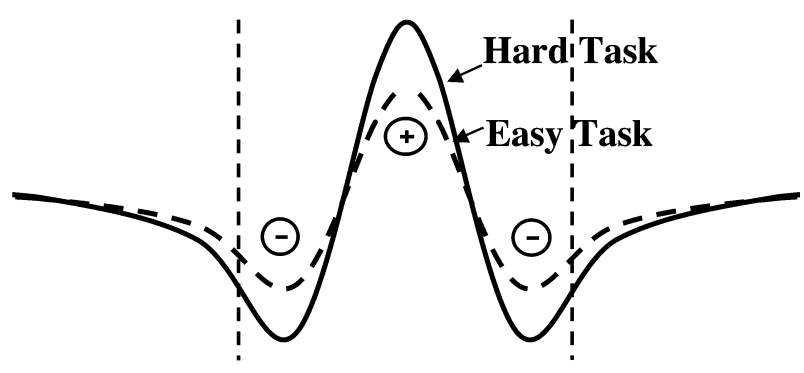

Figure 5. A push-pull mechanism of spatial selective attention. Simplified model of a mechanism that may account for some of the reviewed findings. It is proposed that attention facilitates visual information processing at the attended location $(+)$ and, at the same time, suppresses information processing at unattended locations $(-)$. The suppressive mechanisms operate most strongly at the level of the receptive field (RF). Additional extra-RF mechanisms mediate the filtering of unwanted information over large spatial scales. The degree to which this interdependent push-pull mechanism can be activated depends on the attentional demands of the task at hand. An attentionally demanding hard task will activate both the facilitatory and the suppressive mechanisms more strongly than a less attentionally demanding easy task will. 
of visual information. Alternatively, in accord with the model, this result can be explained by the smaller RFs and less extensive extra-RF surrounds of the early processing stages (see also Kastner et al., 1998; Kastner et al., 2001). Second, the effects of attentional response enhancement and suppression were mirroring each other at intermediate processing levels, which can be explained by the coactivation of the faciltatory and the suppressive mechanisms in the model. And third, the magnitude of the attentional response enhancement and suppression depended on the attentional load, which is reflected in the model by the lateral extent to which the suppressive mechanisms can be activated and by the variable amplitude of the facilitatory mechanism. In summary, the facilitation in the processing of selected information and the filtering of unwanted information may be mediated by an attention mechanism that operates in a push-pull fashion at intermediate processing levels of the visual cortex. Such a mechanism is likely controlled by a distributed network of higher order areas, as will be described in the next section.

\section{Frontal and Parietal Cortex: \\ Sorting Out Sources and Sites}

There is evidence from studies of patients suffering from attentional deficits due to brain damage and from functional brain imaging studies of healthy subjects performing attention tasks that attention-related modulatory signals are not generated within the visual system but, rather, derive from higher order areas in the parietal and frontal cortex and are transmitted via feedback projections to the visual system (Corbetta \& Shulman, 2002; Kanwisher \& Wojciulik, 2000; Kastner \& Ungerleider, 2000; Nobre, 2001). For example, in our fMRI studies, activations of the parietal and frontal cortex were investigated in addition to activations within the visual cortex. Results for a single subject are shown in Figure 6A. In this subject, the FEFs were activated bilaterally, together with the supplementary eye field (SEF) and the superior parietal lobule (SPL) during directed attention to the periphery. A network consisting of areas in the SPL, the FEFs, and the SEF was consistently activated across subjects and has been found to be activated in a variety of visuospatial attention tasks (for meta-analyses, see Kastner \& Ungerleider, 2000; Pessoa, Kastner, \& Ungerleider, 2002). Thus, there appears to be a general attention network that operates independently of the specific requirements of the visuospatial attention task. However, from these studies, it is not clear whether the activity found in areas of the frontal and parietal cortex reflects complex processing of visual information or whether this activity reflects the attentional operations themselves. To sort out sources that generate attentional feedback signals from sites that receive top-down modulatory signals, we performed an experiment that probed the effects of spatially directed attention in the presence and in the absence of visual stimulation (Kastner, Pinsk, et al., 1999).
A third experimental condition was added to the design that was used to investigate competitive interactions and their modulation by spatial attention, as has been described above. In addition to the two visual presentation conditions, sequential and simultaneous, and the two attentional conditions, unattended and attended, an expectation period preceding the attended presentations was introduced, during which subjects were required to direct attention covertly to the target location and were instructed to expect the occurrences of the stimulus presentations. In this way, the effects of attention in the presence (ATT in Figures 6C and 6D) and absence (EXP in Figures $6 \mathrm{C}$ and $6 \mathrm{D}$ ) of visual stimulation could be studied. The time courses of fMRI signals in activated regions of visual, frontal, and parietal cortex were analyzed to dissociate sources of attentional top-down feedback and sites of attentional modulation.

In the visual cortex, as illustrated for area V4 in Figure $6 \mathrm{C}$, the fMRI signals increased during the expectation period (textured blocks), before any stimuli were present on the screen. This increase of baseline activity was followed by a further increase of activity evoked by the onset of the stimulus presentations (gray shaded blocks). The baseline increase was found in all visual areas with a representation of the attended location, indicating that it was topographically specific. It was strongest in V4 but was also seen in early visual areas. It is noteworthy that baseline increases were found in V1, even though no significant attentional modulation of visually evoked activity was seen in this area in this experiment.

These results from the visual cortex are in accordance with single-cell recording studies showing that spontaneous (baseline) firing rates were $30 \%-40 \%$ higher for neurons in areas V2 and V4 when the animal was cued to attend covertly to a location within the neuron's RF before the stimulus was presented there (Luck et al., 1997). The baseline increases found in the human visual cortex may be subserved by increases in spontaneous firing rate similar to those found in these physiology studies but summed over large populations of neurons. Baseline increases have been shown to depend on the anticipated task difficulty (Ress, Backus, \& Heeger, 2000). The increases evoked by directing attention to a target location in anticipation of a behaviorally relevant stimulus are likely to reflect a top-down feedback bias in favor of the attended location in the human visual system.

In the parietal and frontal cortex, the same distributed network for spatial attention was activated during directed attention in the absence of visual stimulation as during directed attention in the presence of visual stimulation, consisting of the FEF, the SEF, and the SPL (Figures $6 \mathrm{~A}$ and $6 \mathrm{~B}$ ). A time course analysis of the fMRI signals revealed that, as in visual cortical areas, there was an increase in activity in these frontal and parietal areas, due to directed attention in the absence of visual input. However, first, this increase in activity was stronger in the SPL, the FEF, and the SEF than the increase in activity seen in the visual cortex (as exemplified for the FEF in Fig- 
A

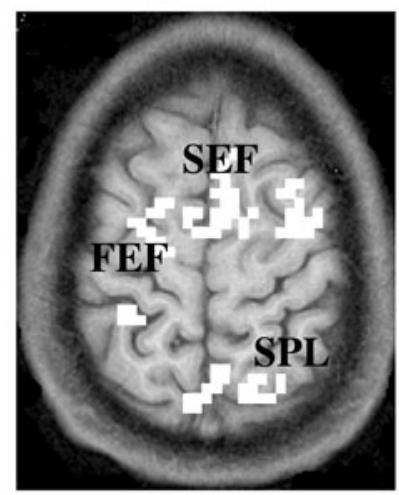

Visual Stimuli and Attention

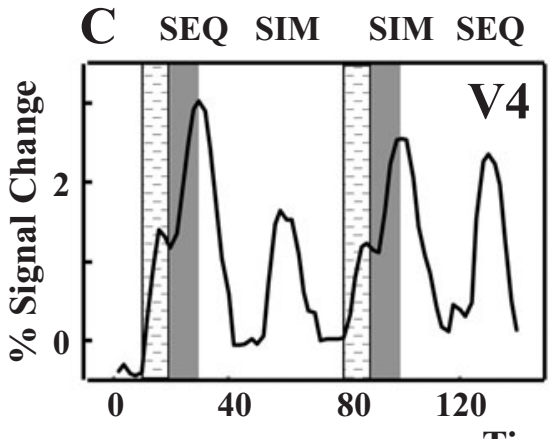

B

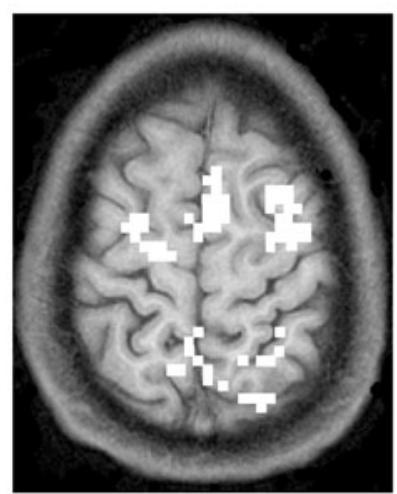

Attention Without Visual Stimuli
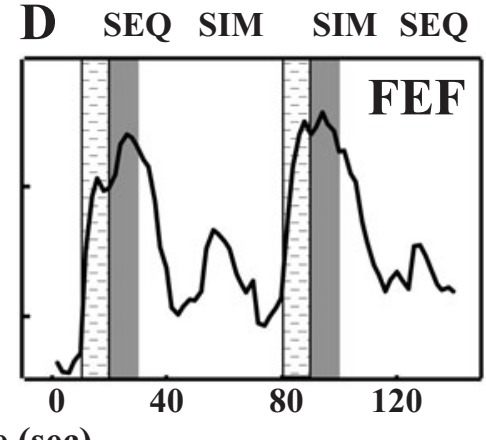

EXP

\section{ATT}

Figure 6. A fronto-parietal network for spatial attention. Axial slice through the frontal and parietal cortex. (A) When the subject directed attention to a peripheral target location and performed a discrimination task, a distributed fronto-parietal network was activated including the supplementary eye field (SEF), the frontal eye field (FEF), and the superior parietal lobule (SPL). (B) The same network of frontal and parietal areas was activated when the subject directed attention to the peripheral target location in expectation of the stimulus onset. (C) Time series of fMRI signals in V4. Directing attention to a peripheral target location in the absence of visual stimulation led to an increase of baseline activity (EXP, textured blocks), which was followed by a further increase after the onset of the stimuli (ATT, gray shaded blocks). Baseline increases were found in both the striate and the extrastriate visual cortex. (D) Time series of fMRI signals in the FEF. Directing attention to the peripheral target location in the absence of visual stimulation led to a stronger increase in baseline activity than in the visual cortex; the further increase of activity after the onset of the stimuli was not significant.

ure 6D), and second, there was no further increase in activity evoked by the attended stimulus presentations in these parietal and frontal areas. Rather, there was sustained activity throughout the expectation period and the attended presentations (Figure 6D).

These results from parietal and frontal areas suggest that the activity reflected the attentional operations of the task, and not visual processing. These findings therefore provide the first evidence that these parietal and frontal areas may be the sources of the feedback that generated the top-down biasing signals seen in the visual cortex. The anatomical connections of the SPL, FEF, and SEF put them in a position to serve as sources of top-down feedback signals that modulate neural processing in the visual system. In the monkey, the FEF and SEF are reciprocally connected with ventral stream areas (Ungerleider, Gaffan, \& Pelak, 1989; Webster, Bachevalier, \& Ungerleider, 1994) and the posterior parietal cortex (Cavada \& Goldman-Rakic, 1989). The posterior parietal cortex is connected with ventral stream areas via the lateral intraparietal area (area LIP; Webster et al., 1994).

A functional role for the fronto-parietal attention network in attentional control was further suggested in the study that probed target search related and distractor- 
related activity, described above (Pinsk et al., 2004). In areas of the fronto-parietal attention network, activity increased with increasing attentional load, thereby reflecting the subjects' behavioral performance. In this respect, higher order areas showed similar response patterns of target search related activity, as compared with extrastriate areas V4 and TEO (see Figure 4, "Target-Related Activity"). However, unlike in these extrastriate areas, activity was entirely suppressed in the fronto-parietal network when the subjects performed the fixation task and ignored target stimuli. Hence, areas of the frontoparietal attention network were activated only when target stimuli were attentionally selected, but not when the same stimuli were ignored, supporting the idea that these areas are involved with target selection and distractor suppression during spatially directed attention, rather than sensory-driven processes (Everling et al., 2002; Moore \& Armstrong, 2003; Schall \& Thompson, 1999).

In summary, a distributed network of areas in the frontal and parietal cortex appears to be the source of the attentional modulation obtained in the visual cortex. Although much needs to be learned about the functional roles of the different parts of this network in attentional control, as a whole, the network mediates target selection and distractor suppression.

\section{The Pulvinar: An Integrator and Coordinator in the Thalamus?}

Several lines of evidence indicate that the pulvinar, located in the dorsal thalamus, is also part of the distributed network subserving visuospatial attention (Robinson \& Petersen, 1992; Shipp, 2004). First, when the pulvinar is inactivated by muscimol, a GABA agonist, monkeys show impairments in filtering out irrelevant information from distractor stimuli (Desimone, Wessinger, Thomas, \& Schneider, 1990). A similar role of the pulvinar in filtering unwanted information has also been suggested by neuroimaging studies in humans (LaBerge \& Buchsbaum, 1990). And second, both patients with lesions in the pulvinar and monkeys with an inactivated pulvinar exhibit attentional deficits, such as impairments in directing attention to the contralateral hemifield (Karnath, Himmelbach, \& Rorden, 2002; Petersen, Robinson, \& Morris, 1987; Rafal \& Posner, 1987). A similar role of the pulvinar in mediating shifts of attention has also been suggested by neuroimaging studies of normal human subjects (Yantis et al., 2002). As we will review below, on the basis of its anatomical connectivity and functional organization, it is possible that the pulvinar may operate by integrating visual information and coordinating attentional function in concert with the fronto-parietal network. However, since the functions of the pulvinar are generally not well understood, future research will be needed to support the ideas about a possible role of the pulvinar as a thalamic integrator and coordinator of attentional function.

Anatomical and functional organization of visual maps in the pulvinar. In the macaque monkey, the pulvinar contains in its inferior, lateral, and medial subdivi- sions at least four visual areas that can be distinguished on the basis of their visuotopic organization and connectivity with striate and extrastriate visual areas (Maunsell \& Van Essen, 1983; Standage \& Benevento, 1983; Ungerleider, Desimone, Galkin, \& Mishkin, 1984; Ungerleider, Galkin, \& Mishkin, 1983). Two of these areas are located in the inferior and lateral part of the pulvinar. These areas contain clearly organized retinotopic maps and are reciprocally connected with cortical areas V1, V2, V4, and MT. Both areas also receive inputs from the superficial layers of the superior colliculus (SC), which is part of a distributed network of areas controlling eye movements (Benevento \& Standage, 1983; Harting, Huerta, Frankfurter, Strominger, \& Royce, 1980). A third area, described on the basis of its connections with dorsal extrastriate areas MT, MST, and FST, is located in the medial portion of the inferior subdivision and does not appear to have a well-defined retinotopic map (Ungerleider et al., 1983, Ungerleider et al., 1984). A fourth area, known as the Pdm, has been identified dorsal to these areas and also does not show much visuotopic organization. The Pdm receives input from the SC (Benevento \& Standage, 1983); its interconnections with the cortex are unknown.

The functions of the pulvinar are not well understood. It has been shown that the majority of neurons in all the visual areas of the pulvinar are responsive to a variety of visual stimulus properties, such as orientation or direction of movement, but without much specificity (Petersen, Robinson, \& Keys, 1985). Neural responses in these areas can also be influenced by eye movements and eye position (Robinson, McClurkin, \& Kertzman, 1990; Robinson, Petersen, \& Keys, 1986), which is likely related to the SC inputs on the pulvinar and suggests a functional role in the network for the control of eye movements. A specific role in selective visual attention has been proposed for the Pdm. In this area, neural responses were enhanced when an animal attended to a visual stimulus, as compared with when the same stimulus was ignored (Petersen et al., 1985). Importantly, the enhancement effects were seen only when the monkey attended to a stimulus in the RF, but not when it attended to a simultaneously presented stimulus elsewhere in the visual field, indicating the spatial selectivity of the attention effects. At the behavioral level, inactivation of the Pdm by injecting a GABA agonist led to impairments in directing attention to the contralateral hemifield, whereas injections of a GABA antagonist induced a facilitation of the allocation of attention (Petersen et al., 1987).

In addition to the functional evidence described thus far, a role for the pulvinar as a thalamic integrator and coordinator of attentional function has been suggested on the basis of its unique anatomical connectivity (e.g., Shipp, 2004). First, the visual maps in areas of the pulvinar are organized in such a way that neurons representing corresponding parts of the visual field in cortical visual areas project to similar parts of the pulvinar maps (Adams, Hof, Gattass, Webster, \& Ungerleider, 2000; Shipp, 2001, 2003). Thereby, visual information from different cortical areas could be integrated in pul- 
vinar areas. Indeed, striate and extrastriate areas might use the pulvinar maps for indirect transcortical communication that may complement and extend local corticocortical feedback circuits. Evidence in support of such an idea has been found in single-cell physiology studies demonstrating that pulvinar neurons integrate both object and spatial properties of visual stimuli that are represented in separate cortical areas (Benevento \& Port, 1995). Second, areas of the fronto-parietal attention network - namely, the FEF and the LIP area - have reciprocal connections with the $\mathrm{SC}$, which also projects to visual areas of the pulvinar (Benevento \& Standage, 1983; Harting et al., 1980). Hence, this cortico-colliculopulvinar loop provides a link between the fronto-parietal attention network and the pulvinar that could play an important role in coordinating attentional control functions (Shipp, 2004), which may be exerted in concert by cortical and subcortical networks, as summarized in the neural architecture diagram depicted in Figure 7.

Results from human neuroimaging studies. In recent fMRI studies, we performed a series of experiments that were aimed at identifying a homologue for area Pdm in the human pulvinar (Kastner et al., 2004). Flickering checkerboard stimuli were presented simultaneously to both hemifields, interleaved with blank periods, or unilaterally in alternation to the right or the left hemifield. In one set of studies, the subjects were instructed by an arrow presented at fixation to covertly direct attention to either the left or the right checkerboard and to detect luminance changes that occurred at random times and at $10^{\circ}$ eccentricity. In another set of studies, the subjects were instructed to maintain fixation and to passively view the stimuli without performing any specific task.

During bilateral attended presentations of the checkerboard stimuli, two regions within the pulvinar were consistently activated across subjects and were found to be located superior and medial to the human LGN. The region in the right pulvinar is depicted for an individual subject in Figure 8A. Given the individual variability of the locations of activated regions within the pulvinar, it is not clear whether the regions in the left and the right pulvinar represent corresponding visual areas. Importantly, our attention-related activity in dorso-medial regions of the human pulvinar was located in the same region as the lesion site in neglect patients (Karnath et al., 2002). When subjects directed attention to checkerboard

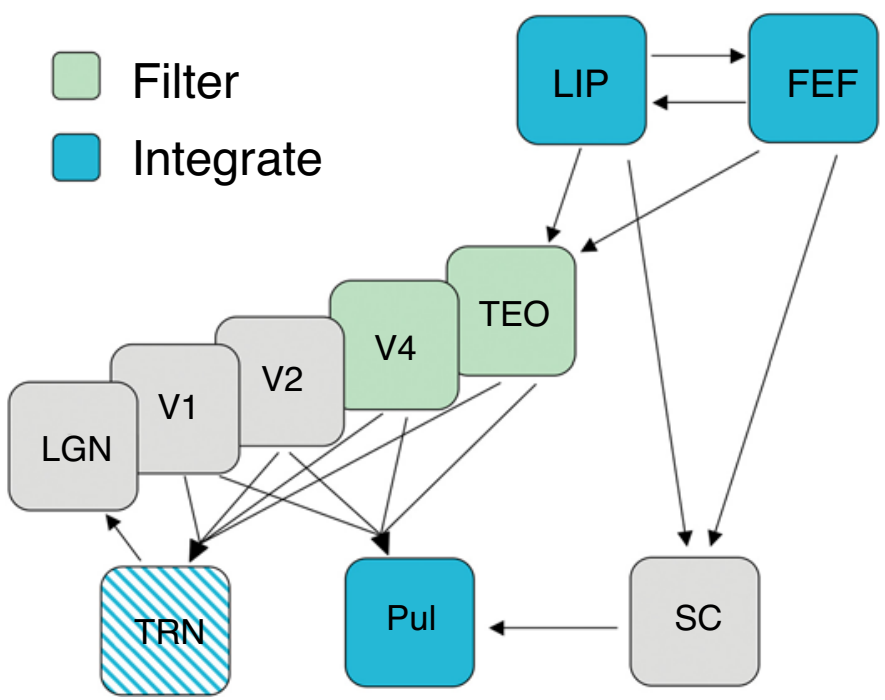

Figure 7. Neural architecture of visual attention. The schematic diagram illustrates the widely distributed networks of brain areas that subserve visual attention and operate across various processing levels. The lateral geniculate nucleus (LGN) is the first stage at which visual processing is modulated by attention; this modulation may be under control of the thalamic reticular nucleus (TRN), which operates as a local integrator of visual information (striped blue box). Intermediate cortical areas V4 and TEO act as filter sites to reduce the amount of unwanted information (green boxes). Higher order areas in the parietal (LIP) and frontal (FEF) cortices integrate information from the visual system and provide top-down attentional control via feedback connections (blue boxes). Furthermore, the pulvinar (Pul) may act as an additional integrator receiving information from both the visual system and the higher order areas via the superior colliculus (SC). The connectivity of these brain systems is indicated in simplified form and does not reflect the complexity of the known anatomical connections. It should be noted that most, if not all, of the connections are reciprocal. 


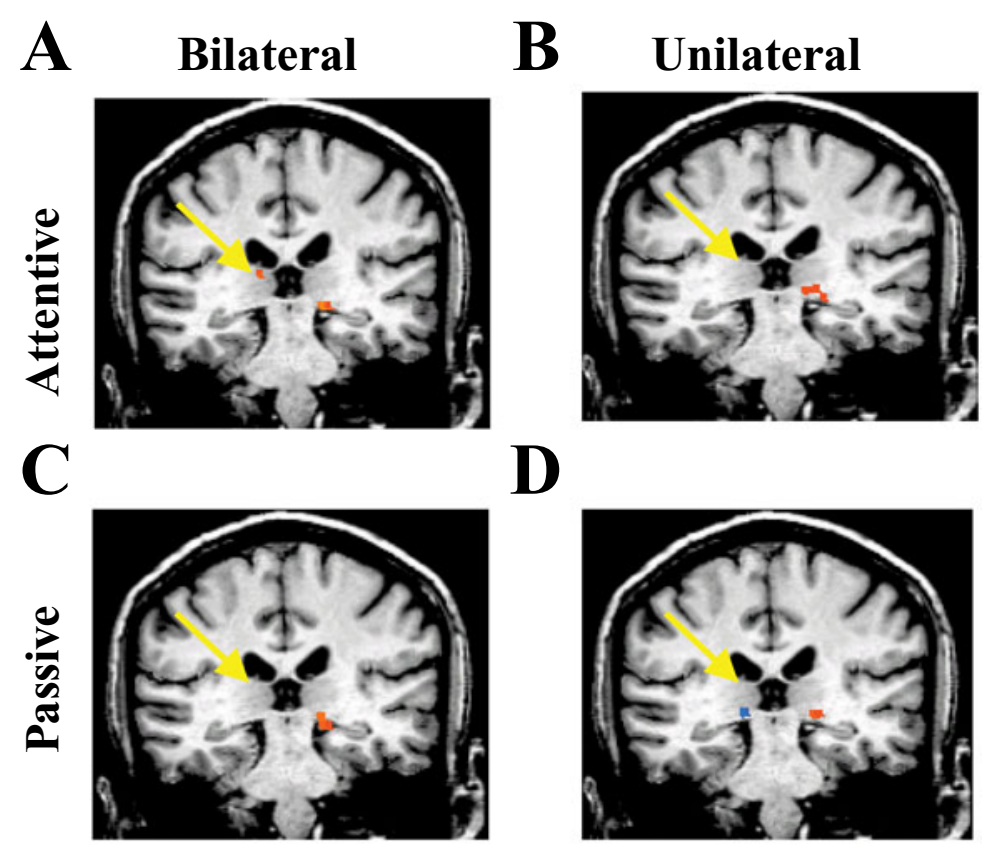

Figure 8. Functional activation of the pulvinar during directed attention and passive viewing. Results are from 1 subject tested in four different experiments. (A) Bilateral attentive. The arrow indicates functional activation of the right pulvinar. The subject directed attention to a checkerboard stimulus presented simultaneously to the right and left visual hemifields. (B) Unilateral attentive. No functional activation was found in the pulvinar when the stimuli were presented unilaterally and in alternation to the right and left hemifields while the subjects directed attention to the checkerboards. (C) Bilateral passive. No functional activation was obtained in the pulvinar when the stimuli were presented bilaterally and were passively viewed by the subjects while maintaining fixation. (D) Unilateral passive. No functional activation was found in the pulvinar when the stimuli were presented in alternation to the right or the left hemifield and were passively viewed by the subjects while maintaining fixation. From "Functional Imaging of the Human Lateral Geniculate Nucleus and Pulvinar," by S. Kastner, D. H. O'Connor, M. M. Fukui, H. M. Fehd, U. Herwig, and M. A. Pinsk, 2004, Journal of Neurophysiology, 91, p. 443. Copyright 2004 by the American Physiological Society. Reprinted with permission.

stimuli presented unilaterally in alternation to the right and the left hemifields, no activations in the pulvinar were found (Figure 8B). Because our statistical analyses contrasted presentations to one hemifield with presentations to the other hemifield, it is possible that similar regions in the pulvinar were activated in both cases and, therefore, subtracted out in the analysis. This finding may therefore indicate that visually responsive neurons in the activated region of the pulvinar had large RFs extending over the entire visual field. To test the possibility that the visually evoked pulvinar activity was driven by selective attention, rather than by sensory input, the same experiments were performed while the subjects did not direct attention to the checkerboard stimuli but, rather, passively viewed the stimuli while maintaining fixation. No activation in the pulvinar was found under these conditions, as is illustrated in Figure 8C for the bilateral presentations and in Figure 8D for the unilateral presentations. Thus, as in areas of the fronto-parietal network, the pulvinar activity appeared to be driven by the attentional operations themselves, rather than by sensory input.

Several of our findings support the idea that the activated regions in the left and right dorso-medial pulvinar may be homologous to area Pdm of the macaque pulvinar. First, the human pulvinar areas were strongly modulated by visuospatial attention. Such attentional modulation has been described for the Pdm, but not for other visual areas of the macaque pulvinar (Petersen et al., 1985). Second, the human pulvinar areas appeared to have a coarse visuotopic organization with large RFs extending over the entire visual field. Neurons with large RFs of $>30^{\circ}$ extending across the vertical meridian have been found in the macaque Pdm, but not in other pulvinar areas (Petersen et al., 1985). And third, it is unlikely that the human pulvinar activations occurred in retinotopically organized pulvinar areas, because they were not evoked by alternating hemifield stimuli. It is not clear why retinotopically organized areas of the human pul- 
vinar were not activated in our studies. However, given the small size of the activated volumes within the pulvinar, this may reflect a lack of sensitivity and spatial resolution of the fMRI methods used in our studies. Future studies in which improved spatial resolution will be used will hopefully provide further insights into pulvinar function. Taken together, our findings support the notion of a functional role of the pulvinar in visuospatial attention, in accordance with previous studies that have emphasized its functions in the filtering of unwanted information (Desimone et al., 1990; LaBerge \& Buchsbaum, 1990 ) and in mediating shifts of attention across the visual field (Yantis et al., 2002).

In summary, on the basis of its anatomical connectivity, the pulvinar of the thalamus appears to be in a unique position to operate both as an integrator of visual information by virtue of the registered visual topographic maps of cortico-pulvino-cortical circuits and as a coordinator of attentional function by virtue of its link to the fronto-parietal attention network via a cortico-colliculopulvinar loop. Studies aimed at elucidating particularly the cortical connectivity of the Pdm and probing more complex function using simultaneous recordings from multiple sites of the cortical and subcortical attention network in behaving monkeys will be needed to provide the experimental evidence in support of these ideas.

\section{Conclusions: Neural Architecture of Visual Attention}

Evidence from functional brain imaging reveals that attention operates at various processing levels within the visual system and beyond. First, the LGN appears to be the first stage in the processing of visual information that is modulated by attention, consistent with the idea that it may play an important role as an early gatekeeper in controlling neural gain. Second, areas at intermediate cortical processing levels, such as V4 and TEO, appear to be important sites at which attention filters out unwanted information by means of intra- and extra-RF mechanisms. These attention mechanisms operate in a push-pull fashion to facilitate the processing of attended stimuli and to suppress the processing of unattended stimuli. Third, the attention mechanisms that operate in the visual system appear to be controlled by a distributed network of higher order areas in the frontal and parietal cortex, which generate top-down signals that are transmitted via feedback connections to the visual system. Fourth, the pulvinar may serve as a subcortical integrator of visual information and a coordinator of attentional control functions, in concert with the fronto-parietal network. The complex neural architecture of this multilevel attention network is summarized in Figure 7. It should be noted that the pattern of connectivity depicted in Figure 7 is schematic and, therefore, presents a simplification of known anatomical connections. Furthermore, we have just begun to understand some of the attentional functions associated with the different parts of this neural architecture, and our assumptions about functionality are held cautiously. Together, these widely distributed brain systems cooperate to generate attention functions for the selection of behaviorally relevant information that can be further utilized in other cognitive networks to ultimately guide goal-directed action.

\section{REFERENCES}

Adams, M. M., Hof, P. R., Gattass, R., Webster, M. J., \& UngerleiDER, L. G. (2000). Visual cortical projections and chemoarchitecture of macaque monkey pulvinar. Journal of Comparative Neurology, 419, 377-393.

BAHCALl, D. O., \& Kowler, E. (1999). Attentional interference at small spatial separations. Vision Research, 39, 71-86.

Benevento, L. A., \& PorT, J. D. (1995). Single neurons with both form/color differential responses and saccade-related responses in the nonretinotopic pulvinar of the behaving macaque monkey. Visual Neuroscience, 12, 523-544.

BENEVENTO, L. A., \& STANDAGE, G. P. (1983). The organization of projections of the retinorecipient and nonretinorecipient nuclei of the pretectal complex and layers of the superior colliculus to the lateral pulvinar and medial pulvinar in the macaque monkey. Journal of Comparative Neurology, 217, 307-336.

BREFCZYNSKI, J. A., \& DEYOE, E. A. (1999). A physiological correlate of the "spotlight" of visual attention. Nature Neuroscience, 2, 370374.

Broadbent, D. (1958). Perception and communication. London: Pergamon.

Cameron, E. L., TaI, J. C., \& Carrasco, M. (2002). Covert attention affects the psychometric function of contrast sensitivity. Vision Research, 42, 949-967.

CAPUTO, G., \& GUERRA, S. (1998). Attentional selection by distractor suppression. Vision Research, 38, 669-689.

Carrasco, M., LING, S., \& READ, S. (2004). Attention alters appearance. Nature Neuroscience, 7, 308-313.

Cavada, C., \& Goldman-RaKic, P. S. (1989). Posterior parietal cortex in rhesus monkey: II. Evidence for segregated corticocortical networks linking sensory and limbic areas with the frontal lobe. Journal of Comparative Neurology, 287, 422-445.

CAVE K. R., \& ZIMMERMAN J. M. (1997). Flexibility in spatial attention before and after practice. Psychological Science, 8, 399-403.

Chelazzi, L., Duncan, J., Miller, E. K., \& Desimone, R. (1998). Responses of neurons in inferior temporal cortex during memory-guided visual search. Journal of Neurophysiology, 80, 2918-2940.

Chen, W., Zhu, X. H., Thulborn, K. R., \& UGURbil, K. (1999). Retinotopic mapping of lateral geniculate nucleus in humans using functional magnetic resonance imaging. Proceedings of the National Academy of Sciences, 96, 2430-2434.

CHEng, A., Eysel, U. T., \& Vidyasagar, T. R. (2004). The role of the magnocellular pathway in serial deployment of visual attention. European Journal of Neuroscience, 20, 2188-2192.

CoOK, E. P., \& MAUnSELL, J. H. (2002). Attentional modulation of behavioral performance and neuronal responses in middle temporal and ventral intraparietal areas of macaque monkey. Journal of Neuroscience, 22, 1994-2004.

Corbetta, M., Miezin, F. M., Dobmeyer, S., Shulman, G. L., \& PeTERSEN, S. E. (1990). Attentional modulation of neural processing of shape, color, and velocity in humans. Science, 248, 1556-1559.

Corbetta, M., \& Shulman, G. L. (2002). Control of goal-directed and stimulus-driven attention in the brain. Nature Reviews Neuroscience, 3, 201-215.

CRICK, F. (1984). Function of the thalamic reticular complex: The searchlight hypothesis. Proceedings of the National Academy of Sciences, 81, 4586-4590.

CUTzu, F., \& Tsotsos, J. K. (2003). The selective tuning model of attention: Psychophysical evidence for a suppressive annulus around an attended item. Vision Research, 43, 205-219. 
Desimone, R., \& Duncan, J. (1995). Neural mechanisms of selective visual attention. Annual Review of Neuroscience, 18, 193-222.

Desimone, R., Moran, J., Schein, S. J., \& Mishkin, M. (1993). A role for the corpus callosum in visual area V4 of the macaque. Visual Neuroscience, 10, 159-171.

DESIMONE R., \& UNGERLEIDER L. G. (1989). Neural mechanisms of visual processing in monkeys. In F. Boller \& J. Grafam (Eds.), Handbook of neuropsychology (Vol. 2, pp. 267-299). Amsterdam: Elsevier.

Desimone, R., Wessinger, M., Thomas, L., \& Schneider, W. (1990). Attentional control of visual perception: Cortical and subcortical mechanisms. Cold Spring Harbor Symposia on Quantitative Biology. 55, 963-971.

DeWEerd, P., Peralta, M. R., III, Desimone, R., \& Ungerleider, L. G (1999). Loss of attentional stimulus selection after extrastriate cortical lesions in macaques. Nature Neuroscience, 2, 753-758.

Duncan, J. (1996). Cooperating brain systems in selective perception and action. In T. Inui \& J. L. McClelland (Eds.), Attention and performance XVI (pp. 549-578). Cambridge, MA: MIT Press.

ERIKSEN, C. W., \& ST. JAMES, J. D. (1986). Visual attention within and around the field of focal attention: A zoom lens model. Perception \& Psychophysics, 40, 225-240.

ERISIR, A., VAN HORN, S. C., \& Sherman, S. M. (1997). Relative numbers of cortical and brainstem inputs to the lateral geniculate nucleus. Proceedings of the National Academy of Sciences, 94, 1517-1520.

Everling, S., Tinsley, C. J., Gaffan, D., \& Duncan, J. (2002). Filtering of neural signals by focused attention in the monkey prefrontal cortex. Nature Neuroscience, 5, 671-676.

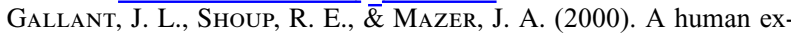 trastriate area functionally homologous to macaque V4. Neuron, 27, 227-235.

GoldMAN-RAKIC, P. S. (1988). Topography of cognition: Parallel distributed networks in primate association cortex. Annual Review of Neuroscience, 11, 137-156.

GuILlery, R. W., FEIG, S. L., \& Lozsádi, D. A. (1998). Paying attention to the thalamic reticular nucleus. Trends in Neurosciences, $\mathbf{2 1}$ 28-32.

Harting, J. K., Huerta, M. F., Frankfurter, A. J., Strominger, N. L., \& RoyCE, G. J. (1980). Ascending pathways from the monkey superior colliculus: An autoradiographic analysis. Journal of Comparative neurology, 192, 853-882.

Ito, M., \& GilberT, C. D. (1999). Attention modulates contextual influences in the primary visual cortex of alert monkeys. Neuron, 22 593-604.

JoNES, E. G. (1985). The thalamus. New York. Plenum.

KANWISHer, N., \& WoJCIULIK, E. (2000). Visual attention: Insights from brain imaging. Nature Reviews Neuroscience, 1, 91-100.

Karnath, H. O., Himmelbach, M., \& Rorden, C. (2002). The subcortical anatomy of human spatial neglect: Putamen, caudate nucleus and pulvinar. Brain, 125(Pt. 2), 350-360.

KASTNER, S. (2004). Towards a neural basis of human visual attention: Evidence from functional brain imaging. In J. Duncan \& N. Kanwisher (Eds.), Attention and performance XX (pp. 299-318). Oxford: Oxford University Press.

Kastner, S., DeWeerd, P., Desimone, R., \& Ungerleider, L. G. (1998). Mechanisms of directed attention in the human extrastriate cortex as revealed by functional MRI. Science, 282, 108-111.

Kastner, S., DeWeerd, P., PinsK, M. A., Elizondo, M. I., Desimone, R., \& UNGERLEIDER, L. G. (2001). Modulation of sensory suppression: Implications for receptive field sizes in the human visual cortex. Journal of Neurophysiology, 86, 1398-1411.

Kastner, S., Nothdurft, H. C., \& Pigarev, I. N. (1999). Neuronal responses to orientation and motion contrast in cat striate cortex. Visual Neuroscience, 16, 587-600

Kastner, S., O'Connor, D. H., Fukui, M. M., Fehd, H. M., HerWIG, U., \& PINSK, M. A. (2004). Functional imaging of the human lateral geniculate nucleus and pulvinar. Journal of Neurophysiology, 91, 438-448.

KAstner, S., Pinsk, M. A., DeWeerd, P., Desimone, R., \& UngerleiDER, L. G. (1999). Increased activity in human visual cortex during directed attention in the absence of visual stimulation. Neuron, $\mathbf{2 2}$ 751-761.
Kastner, S., \& Ungerleider, L. G. (2000). Mechanisms of visual attention in the human cortex. Annual Review of Neuroscience, 23, 315-341.

LABERGE, D., \& Brown, V. (1989). Theory of attentional operations in shape identification. Psychological Review, 96, 101-124.

LaBerge, D., \& Buchsbaum, M. S. (1990). Positron emission tomographic measurements of pulvinar activity during an attention task. Journal of Neuroscience, 10, 613-619.

LaVIE, N., \& Tsal, Y. (1994). Perceptual load as a major determinant of the locus of selection in visual attention. Perception \& Psychophysics, 56, 183-197.

Lu, Z. L., \& Dosher, B. A. (1998). External noise distinguishes attention mechanisms. Vision Research, 38, 1183-1198.

Luck, S. J., Chelazzi, L., Hillyard, S. A., \& Desimone, R. (1997). Neural mechanisms of spatial selective attention in areas V1, V2, and V4 of macaque visual cortex. Journal of Neurophysiology, 77, 2442.

Martinez, A., Anllo-Vento, L., Sereno, M. I., Frank, L. R., Buxton, R. B., Dubowitz, D. J., Wong, E. C., Hinrichs, H., Heinze, H. J., \& Hillyard, S. A. (1999). Involvement of striate and extrastriate visual cortical areas in spatial attention. Nature Neuroscience, 2, 364369.

Maunsell, J. H., \& van Essen, D. C. (1983). The connections of the middle temporal visual area (MT) and their relationship to a cortical hierarchy in the macaque monkey. Journal of Neuroscience, 3, 25632586.

McAdams, C. J., \& Maunsell, J. H. (1999). Effects of attention on orientation-tuning functions of single neurons in macaque cortical area V4. Journal of Neuroscience, 19, 431-441.

Mehta, A. D., Ulbert, I., \& SchroeDER, C. E. (2000). Intermodal selective attention in monkeys: I. Distribution and timing of effects across visual areas. Cerebral Cortex, 10, 343-358.

Merigan, W. H., \& Maunsell, J. H. (1993). How parallel are the primate visual pathways? Annual Review of Neuroscience, 16, 369-402.

Miller, E. K., Gochin, P. M., \& Gross, C. G. (1993). Suppression of visual responses of neurons in inferior temporal cortex of the awake macaque by addition of a second stimulus. Brain Research, 616, 2529.

Moore, T., \& Armstrong, K. M. (2003). Selective gating of visual signals by microstimulation of frontal cortex. Nature, 421, 370-373.

Moran, J., \& Desimone, R. (1985). Selective attention gates visual processing in the extrastriate cortex. Science, 229, 782-784.

MotTer, B. C. (1993). Focal attention produces spatially selective processing in visual cortical areas V1, V2, and V4 in the presence of competing stimuli. Journal of Neurophysiology, 70, 909-919.

MurRaY, S. O., \& WoJCIULIK, E. (2004). Attention increases neural selectivity in the human lateral occipital complex. Nature Neuroscience, 7,70-74.

NoBRE, A. C. (2001). The attentive homunculus: Now you see it, now you don't. Neuroscience \& Biobehavioral Reviews, 25, 477-496.

O'Connor, D. H., FuKui, M. M., Pinsk, M. A., \& KASTNER, S. (2002). Attention modulates responses in the human lateral geniculate nucleus. Nature Neuroscience, 5, 1203-1209.

Pessoa, L., Kastner, S., \& UNGerleider, L. G. (2002). Attentional control of the processing of neural and emotional stimuli. Cognitive Brain Research, 15, 31-45.

PeTERSEN, S. E., RoBinson, D. L., \& Keys, W. (1985). Pulvinar nuclei of the behaving rhesus monkey: Visual responses and their modulation. Journal of Neurophysiology, 54, 867-886.

Petersen, S. E., Robinson, D. L., \& Morris, J. D. (1987). Contributions of the pulvinar to visual spatial attention. Neuropsychologia, 25, 97-105.

PiNSK, M. A., Doniger, G. M., \& Kastner, S. (2004). Push-pull mechanism of selective attention in human extrastriate cortex. Journal of Neurophysiology, 92, 622-629.

PoSNER, M. I. (1980). Orienting of attention. Quarterly Journal of Experimental Psychology, 32, 3-25.

RAFAL, R. D., \& PoSNER, M. I. (1987). Deficits in human visual spatial attention following thalamic lesions. Proceedings of the National Academy of Sciences, 84, 7349-7353.

RECANZONE, G. H., \& WURTZ, R. H. (2000). Effects of attention on MT 
and MST neuronal activity during pursuit initiation. Journal of Neurophysiology, 83, 777-790.

RECANZONE, G. H., WURTZ, R. H., \& SchWARZ, U. (1997). Responses of MT and MST neurons to one and two moving objects in the receptive field. Journal of Neurophysiology, 78, 2904-2915.

ReEs, G., Frith, C. D., \& LAVIE, N. (1997). Modulating irrelevant motion perception by varying attentional load in an unrelated task. Science, 278, 1616-1619.

Rees, G., Wojciulik, E., Clarke, K., Husain, M., Frith, C., \& DriVER, J. (2000). Unconscious activation of visual cortex in the damaged right hemisphere of a parietal patient with extinction. Brain, 123, 1624-1633.

RESS, D., BACKUS, B. T., \& HeEger, D. J. (2000). Activity in primary visual cortex predicts performance in a visual detection task. Nature Neuroscience, 3, 940-945.

ReYnolds, J. H., ChelazZI, L., \& Desimone, R. (1999). Competitive mechanisms subserve attention in macaque areas V2 and V4. Journal of Neuroscience, 19, 1736-1753.

REYNOLDS, J. H., \& DESIMONE, R. (1999). The role of neural mechanisms of attention in solving the binding problem. Neuron, 24, 19-29, 111-125.

Reynolds, J. H., Pasternak, T., \& Desimone, R. (2000). Attention increases sensitivity of V4 neurons. Neuron, 26, 703-714.

Robinson, D. L., McClurkin, J. W., \& KerTZMan, C. (1990). Orbital position and eye movement influences on visual responses in the pulvinar nuclei of the behaving macaque. Experimental Brain Research, 82, 235-246.

RoBINSON, D. L., \& Petersen, S. E. (1992). The pulvinar and visual salience. Trends in Neurosciences, 15, 127-132.

Robinson, D. L., Petersen, S. E., \& KeYs, W. (1986). Saccade-related and visual activities in the pulvinar nuclei of the behaving rhesus monkey. Experimental Brain Research, 62, 625-634.

Roelfsema, P. R., Lamme, V. A., \& SpeKreiJse, H. (1998). Objectbased attention in the primary visual cortex of the macaque monkey. Nature, 395, 376-381.

Schall, J. D., \& THOMPSON, K. G. (1999). Neural selection and control of visually guided eye movements. Annual Review of Neuroscience, 22, 241-259.

SCHNEIDER, K. A., \& KASTNER, S. (2004). Attentional modulation of the human lateral geniculate nucleus and superior colliculus: A highresolution fMRI study. Society for Neuroscience Abstracts, 30, 717.

Sereno, M. I., Dale, A. M., Reppas, J. B., Kwong, K. K., Belliveau, J. W., Brady, T. J., Rosen, B. R., \& Tootell, R. B. (1995). Borders of multiple visual areas in humans revealed by functional magnetic resonance imaging. Science, 268, 889-893.

Sherman, S. M. (2001). Tonic and burst firing: Dual modes of thalamocortical relay. Trends in Neurosciences, 24, 122-126.

Sherman, S. M., \& GUILlery, R. W. (2001). Exploring the thalamus. San Diego: Academic Press.

Sherman, S. M., \& Guillery, R. W. (2002). The role of the thalamus in the flow of information to the cortex. Philosophical Transactions of the Royal Society of London: Series B, 357, 1695-1708.

SHIPP, S. (2001). Corticopulvinar connections of areas V5, V4, and V3 in the macaque monkey: A dual model of retinal and cortical topographies. Journal of Comparative Neurology, 439, 469-490.

SHIPP, S. (2003). The functional logic of cortico-pulvinar connections. Philosophical Transactions of the Royal Society of London: Series B, 358, 1605-1624.

SHIPP, S. (2004). The brain circuitry of attention. Trends in Cognitive Sciences, 8, 223-230.

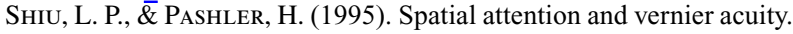 Vision Research, 35, 337-343.

Smith, A. T., SingH, K. D., \& Greenlee, M. W. (2000). Attentional suppression of activity in the human visual cortex. NeuroReport, 11, 271-277.

SPITZER, H., \& Richmond, B. J. (1991). Task difficulty: Ignoring, attending to, and discriminating a visual stimulus yield progressively more activity in inferior temporal neurons. Experimental Brain Research, 83, 340-348.

Standage, G. P., \& Benevento, L. A. (1983). The organization of connections between the pulvinar and visual area MT in the macaque monkey. Brain Research, 262, 288-294.

Steinman, B. A., Steinman, S. B., \& Lehmkuhle, S. (1997). Transient visual attention is dominated by the magnocellular stream. $\mathrm{Vi}$ sion Research, 37, 17-23.

ToOtell, R. B., HADJIKHANi, N., Hall, E. K., Marrett, S., VandufFel, W., Vaughan, J. T., \& Dale, A. M. (1998). The retinotopy of visual spatial attention. Neuron, 21, 1409-1422.

Tsotsos, J. K., Culhane, S. M., \& Cutzu, F. (2001). From foundational principles to a hierarchical selection circuit for attention. In J. Braun, C. Koch, \& J. L. David (Eds.), Visual attention and cortical circuits (pp. 285-306). Cambridge, MA: MIT Press.

Ungerleider, L. G., Desimone, R., Galkin, T. W., \& Mishrin, M. (1984). Subcortical projections of area MT in the macaque. Journal of Comparative Neurology, 223, 368-386.

Ungerleider, L. G., GafFan, D., \& PelaK, V. S. (1989). Projections from inferior temporal cortex to prefrontal cortex via the uncinate fascicle in rhesus monkeys. Experimental Brain Research, 76, 473484.

Ungerleider, L. G., Galkin, T. W., \& Mishin, M. (1983). Visuotopic organization of projections from striate cortex to inferior and lateral pulvinar in rhesus monkey. Journal of Comparative Neurology, 217, 137-157.

Vanduffel, W., Tootell, R. B., \& Orban, G. A. (2000). Attentiondependent suppression of metabolic activity in the early stages of the macaque visual system. Cerebral Cortex, 10, 109-126.

VAn Essen, D. C., Newsome, W. T., \& Bixby, J. L. (1982). The pattern of interhemispheric connections and its relationship to extrastriate visual areas in the macaque monkey. Journal of Neuroscience, 2, 265283.

Vuilleumier, P., Sagiv, N., Hazeltine, E., Poldrack, R. A., Swick, D., RAFAL, R. D., \& GABRIELI, J. D. E. (2001). Neural fate of seen and unseen faces in visuospatial neglect: A combined event-related functional MRI and event-related potential study. Proceedings of the $\mathrm{Na}$ tional Academy of Sciences, 98, 3495-3500.

Webster, M. J., BACHEVAlier, J., \& UNGerLeIDER, L. G. (1994). Connections of inferior temporal areas TEO and TE with parietal and frontal cortex in macaque monkeys. Cerebral Cortex, 4, 470-483.

Wiesel, T. N., \& Hubel, D. H. (1966). Spatial and chromatic interactions in the lateral geniculate body of the rhesus monkey. Journal of Neurophysiology, 29, 1115-1156.

Yantis, S., Schwarzbach, J., Serences, J. T., Carlson, R. L., Steinmetz, M. A., Pekar, J. J., \& Courtney, S. M. (2002). Transient neural activity in human parietal cortex during spatial attention shifts. Nature Neuroscience, 5, 995-1002.

YESHURUN, Y., \& CARRASCO, M. (1998). Attention improves or impairs visual performance by enhancing spatial resolution. Nature, 396, 7275.

Yi, D.-J., Woodman, G. F., Widders, D., Marois, R., \& Chun, M. M. (2004). Neural fate of ignored stimuli: Dissociable effects of perceptual and working memory load. Nature Neuroscience, 7, 992-996.

(Manuscript received August 3, 2004; revision accepted for publication September 30, 2004.) 